\title{
Super-tree Approach for Studying the Phylogeny of Prokaryotes: New Results on Completely Sequenced Genomes
}

\author{
Alexandra Calteau ${ }^{1}$, Vincent Daubin ${ }^{2}$, and Guy Perrieère ${ }^{1}$ \\ 1 Laboratoire de Biométrie et Biologie Évolutive, UMR CNRS 5558, \\ Université Claude Bernard - Lyon 1, 43, bd. Du 11 Novembre 1918, \\ 69622 Villeurbanne Cedex, France \\ \{calteau, perriere\}@biomserv.univ-lyon1.fr \\ http://pbil.univ-lyon1.fr \\ 2 Department of Biochemistry and Molecular Biophysics, \\ 229 Life Sciences South, University of Arizona \\ Tucson, Arizona 85721, USA \\ daubin@email.arizona.edu
}

\begin{abstract}
In this paper we describe a super-tree approach that is able to use the information brought by hundreds of individual gene trees in order to establish a reliable phylogeny of prokaryotes. Indeed, it has been often shown that the use of single genes is not sufficient to solve such a complex case. This is mainly due to problems like reconstruction artifacts, existence of hidden paralogies or the presence of numerous lateral transfers. Here, our super-tree approach allowed us to build two trees containing 86 prokaryotic organisms. All the major bacterial and archeal taxonomic groups are monophyletic in these trees, which present some striking differences with the classical view of prokaryotic phylogeny represented by the ribosomal RNA trees.
\end{abstract}

\section{Introduction}

Many attempts have been made since the precursor work of Woese [1] in order to establish a reliable phylogeny of prokaryotic species (archaea and bacteria). For a long time, the different phylogenies produced using the Small Subunit of ribosomal RNA (SSU rRNA) were considered as the best reference to reveal the evolutionary history of the prokaryotic world [1]-[3]. On these trees, the only well-supported positions were the taxonomic groups located near the base, these groups being represented by hyperthermophilic bacteria, like those from the Thermotoga or Aquifex genera. The lack of resolution between the other divisions was interpreted as the proof of a rapid radiation of the organisms considered. This phenomenon has also been observed in many phylogenies based on protein genes [4]. But improvements realized in methodology led to the conclusion that some parts of Woese's trees were in fact reconstruction artifacts, this especially for the position of hyperthermophiles [5]-[7]. Moreover, it seems that even the supposed very stable rRNA genes could be horizontally transferred 
between species, as some biological experiments suggest [8],[9]. Also, if the hypotheses about the massive occurrence of horizontal transfers between bacterial species are true (see [10]-[14] for many examples), then it seems difficult to use other gene markers to reconstruct the phylogeny of these organisms. Another problem is the fact that a lot of hidden paralogies exist among bacterial protein genes, and therefore risks of obtaining erroneous phylogenies are high when using some markers. Since the availability of an increasing number of complete genomes, several methods have been proposed to build genome-based trees, or to test whether this concept makes sense for bacterial species. The most common approaches are genes concatenation [15]-[17] and orthologous [18] or gene families content [19]-[21] comparisons among species. For concatenation methods, the problem is that the number of genes that are both shared by the different species studied and alignable is very limited (only 23 in the case of [16]). And for the comparison methods, it seems that they are only able to find the relationships between closely related organisms. In that context, we introduced a super-tree method based on the Matrix Representation using Parcimony (MRP) coding scheme [22],[23]. This method already allowed us to produce a tree of life containing 45 species for wich complete genomes were available [24]. At that moment some important bacterial divisions were not represented in our data set (mainly Fusobacteria and Chlorobiales), and incertitudes remained for some parts of the tree. In this paper, we present a more complete study, realized on 86 prokaryotic genomes. The results of our analysis are partially in agreement with the rRNA reference. However, some important differences bring raises questions about bacterial phylogeny.

\section{Material and Methods}

\section{$2.1 \quad$ Gene Families}

A special release of the HOBACGEN database [25] called HOBACGEN-CG was made, gathering all protein sequences into families of homologous genes from the completely sequenced genomes of 76 bacteria and 10 archaea. We retained as orthologous gene families only those containing one gene per species. Though this approach may miss some hidden paralogy, especially in the case of archaea for which only a few organisms are completely sequenced, this definition of orthology has been shown to be much more accurate than a reciprocal BLAST hit based one [26]. Protein sequences from hyperthermophilic bacteria having orthologues only in archaea were removed from the family they belong to since these genes are suspected to have been acquired by lateral transfers [27]-[29]. At last, only families containing at least 30 species were considered for further analysis.

\subsection{Alignments and Gene Trees Construction}

The protein sequences of each family were aligned using CLUSTAL W [30], with all default parameters. To select the parts of the alignments for which homology 
between sites can be assumed with good confidence, we used the GBLOCKS program [31]. It has been shown to give alignments that are almost independent to the different options of CLUSTALÂW. We retained for tree construction only the alignments having conserved at least twice more sites than species. For each family, we computed two trees: one using PHYML [32], a Maximum Likelihood (ML) method, and one using BIONJ [33] an implementation of the NeighbourJoining (NJ) algorithm. For both methods, we used the Jones-Taylor-Thorton (JTT) model of amino acid substitution [34]. In the case of BIONJ trees, the distances were computed using PROTDIST, from the PHYLIP package [35]. Heterogeneities between sites were estimated under a gamma law based model of substitution, and the computation of the alpha parameter was carried out by PUZZLE [36]. All trees were bootstrapped (1000 replicates) using programs SEQBOOT and CONSENSE from PHYLIP.

\subsection{Final Selection of Families}

In order to reduce the impact of inter-domain lateral transfers, we applied the same criteria as in [16], i.e. we screened the trees where bacteria were not monophyletic and we removed these families from the data set or corrected them by removing the transferred sequences from the alignment when it was evident. At last, we ended with 122 trees suitable for super-tree construction.

\subsection{Super-tree Computation}

Trees chosen for the super-tree computation were coded into a binary matrix using the MRP coding scheme [22],[23]. Before we applied this method to complete genomes, it has been used to infer a phylogeny of Eutheria [37]. Each tree obtained for a set of species from a single gene family is coded into a binary matrix of informative sites with respect to bootstrap values as shown in Fig. 1. The matrices obtained are concatenated into a super-matrix in which species absent from a gene family are encoded as unknown state. The super-tree is calculated on the super-matrix using program PAUP* [38] with all default options. 1000 bootstrap replicates are performed on the supermatrix with SEQBOOT and CONSENSE.

\section{Results}

The two super-trees we obtain are quite similar regarding to their topology (Fig. 2 and 3). In both of them, the great bacterial divisions and subdivisions are found to be monophyletic, as well as the Euryarcheota. The lack of resolution of the archaeal part of the tree is due to the low number of genes available for these species. As expected, the bacterial part presents higher bootstrap values and appears thus more resolved, especially with the BIONJ-based trees. The monophyly of Proteobacteria and all their subdivisions, high $\mathrm{G}+\mathrm{C}$ Gram 


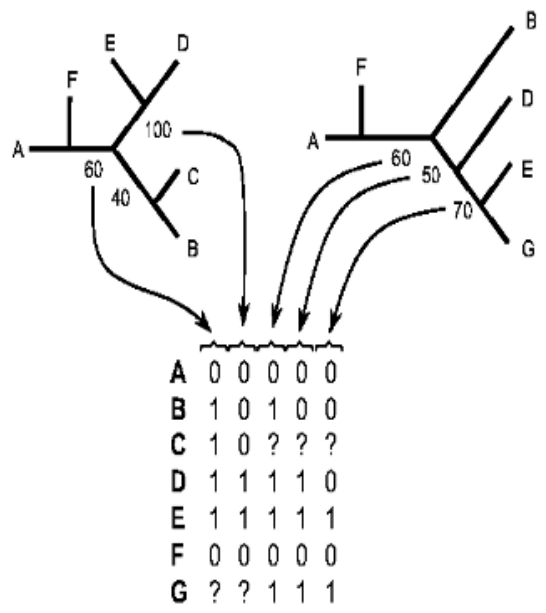

Fig. 1. Construction of super-trees by MRP with bootstrap weighting. Each tree obtained for a set of species from a single orthologous gene family is coded into a binary matrix of informative sites. Only branches having a bootstrap value over $50 \%$ are coded. The matrices obtained are concatenated into a super-matrix in which species absent from a gene family are encoded as unknown state (?). The supertree is computed on the super-matrix with PAUP*.

positives and Spirochetes are strongly supported (bootstrap $\geq 80 \%$ ). The monophyly of low $\mathrm{G}+\mathrm{C}$ Gram positives is also strongly supported in the BIONJ-based super-tree, but has a weaker support in the PHYML-based super-tree. As in our previous study [24], we find that D. Âradiodurans is grouped with the high G+C Gram positives. Also, C.Âtepidum is grouped with the Chlamidiales. Like in the rRNA trees, hyperthermophilic bacteria are located at a basal position, this with a strong support. This is an important difference from our previous results, where this position was occupied by Spirochetes [24]. Here, the Spirochetes are located much deeper in the two super-trees, and this positioning is not supported The remaining parts of the super-trees, and particularly the deep nodes, is not supported. This difficulty of resolving deep branches may be related to the increasing probability of horizontal transfers, hidden paralogies and long branch artifacts with separation time in the individual gene trees.

\section{Discussion - Conclusion}

Although the deep nodes have low support, the level of resolution of the supertree is in strong disagreement with the "genome space" vision of the prokaryotic world predicting a "star phylogeny" [13]. One could argue that grouping of species in the super-tree would only reflect the frequency of gene exchanges between these species. This interpretation can be excluded since the super-tree method would then not be expected to give a tree topology radically different from gene- 


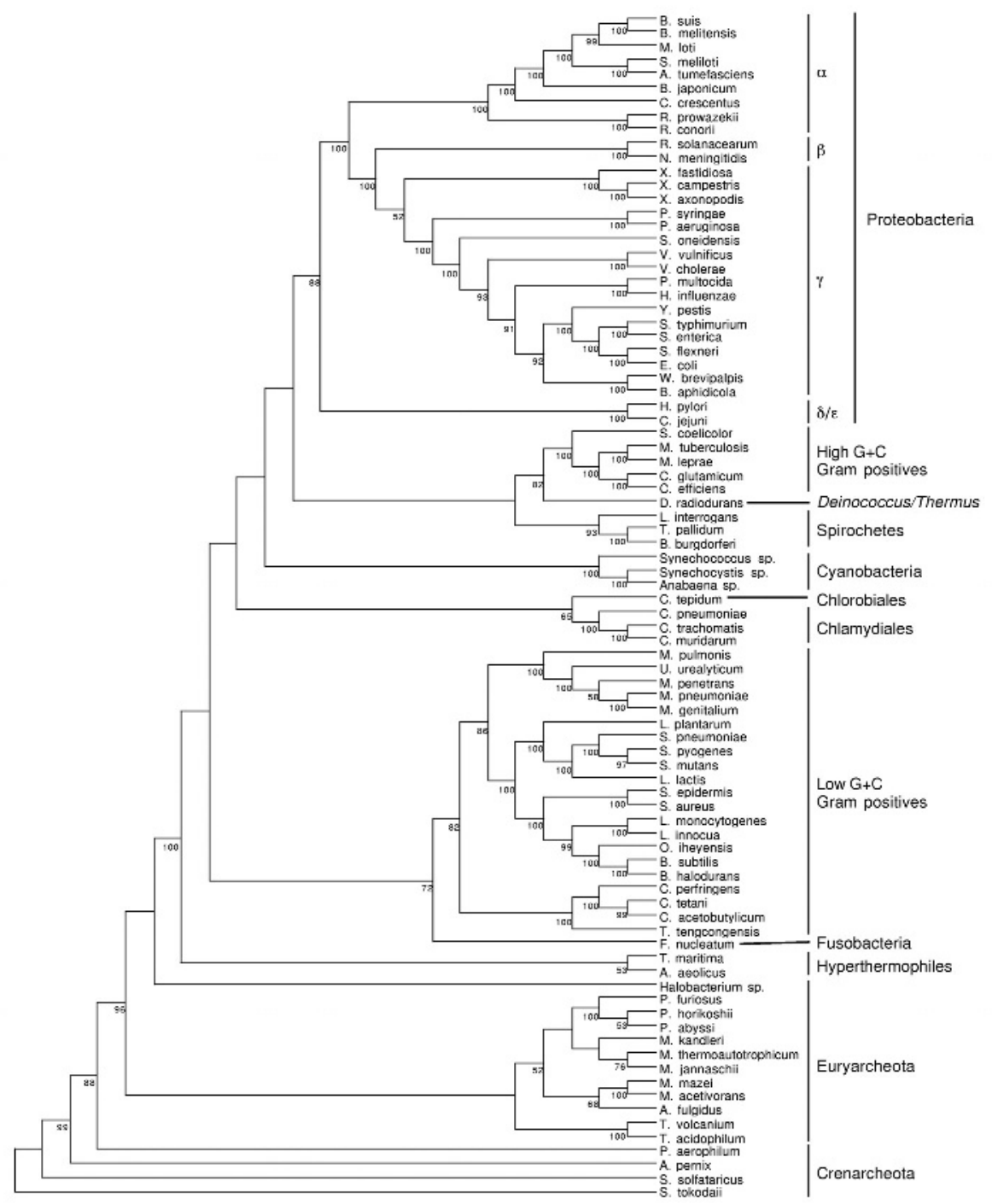

Fig. 2. Super-tree based on 122 gene trees computed with BIONJ. The tree has been arbitrary rooted with $S$. tokodai and only bootstrap values over $50 \%$ are shown.

content based trees [18],[19],[21] which are predicted to be very sensitive to this problem. It is worth to insist on the fact that a particularly stringent selection of protein families was exercised for building the supertree. In particular, a phylogenetic definition of orthology rather than a definition based on reciprocal best 


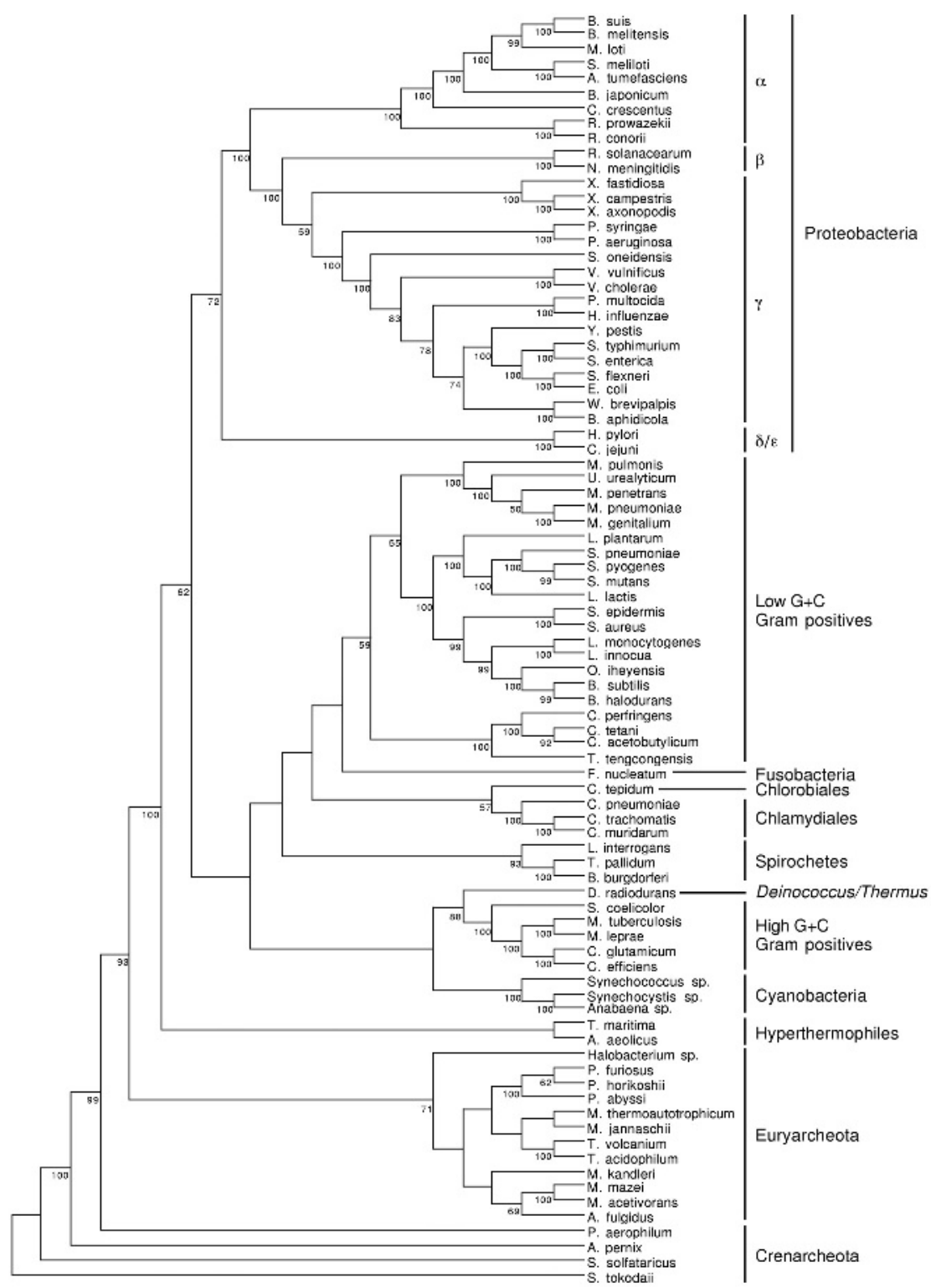

Fig. 3. Super-tree based on 122 gene trees computed with PHYML. The tree has been arbitrary rooted with $S$. tokodai and only bootstrap values over $50 \%$ are shown. 
BLAST hits - as is often the case for practical reasons - was used. Thus, all gene trees where a species was represented more than once were excluded from analysis. This selection allowed to make absolutely no a priori assumption on the topology of the trees, except for the monophyly of bacteria, and to reduce the probability of taking hidden paralogies into account. The phylogeny of Proteobacteria appears is well resolved at this level and is in agreement with the rRNA phylogeny and protein-based works (see[39] for review). Their monophyly (including H.pylori and C.jejunii) is well supported and this last result is particularly valuable because it has rarely been found with genome-tree methods [15],[19],[21]. Equally interesting is the low (or lack of) support for clustering the two hyperthermophilic bacteria, A.aeolicus and T.maritima. Indeed, since proteins of hyperthermophilic bacteria and archaea have been shown to possess a very peculiar amino acid composition [40], it is possible that the grouping of A.aeolicus and T.maritima is linked to a systematic artifact present in the majority of our gene trees. Also, the positioning of these organisms in a basal part of the tree brings us back to a more traditional view of the bacterial world. An explanation for that change could be the presence of horizontally transferred genes in our original data set. Here, due to the fact we had to increase the number of species represented in each family, we mechanically decreased the number of families represented in each species. Therefore, we may have removed horizontally transferred genes responsible for the basal position of Spirochetes, as it was done in [16]. The monophyly of low G+C Gram-positives (including Bacillus and Mycoplasma) on one side, and of high $\mathrm{G}+\mathrm{C}$ Gram-positives on the other side is supported in both super-trees. On the other hand, Gram-positive bacteria are polyphyletic, suggesting that two independent losses of the external membrane occurred in the high- and low $\mathrm{G}+\mathrm{C}$ phyla. The significant support for the position of D.radiodurans is very striking since this organism is usually considered to have a much more basal position among bacteria [1]. This bacterium was already placed there in our previous study [24] and [16] also gives strong support to this position. On the other hand, it remains possible that this position is due to the high $\mathrm{G}+\mathrm{C}$ content of the genome of Deinococcus. Indeed, D.radiodurans is a close relative of Thermus aquaticus, which is a Gram-negative thermophilic bacterium. Though D.radiodurans is positive to the Gram coloration, it has been shown to possess an external membrane unlike Gram-positives. Thus, although this position seems supported, it still needs to be confirmed, in particular by the addition of Thermus in the super-tree.

Acknowledgements. This work has been supported by CNRS and MENRT. A.C. is a recipient of a fellowship from the MENRT.

\section{References}

1. Woese, C.: Bacterial evolution. Microbiol. Rev. 51 (1987) 221-271

2. Barns, S.M., Delwiche, C.F., Palmer, J.D., Pace, N.R.: Perspectives on archaeal diversity, thermophily and monophyly from environmental rRNA sequences. Proc. Natl. Acad. Sci. USA 93 (1996) 9188-9193 
3. Brown, J.R. et Doolittle, W.F.: Archaea and the prokaryote-to-eukaryote transition. Microbiol. Mol. Biol. Rev. 61 (1997) 456-502

4. Koonin, E.V., Makarova, K.S., Aravind, L.: Horizontal gene transfer in prokaryotes: quantification and classification. Annu. Rev. Microbiol. 55 (2001) 709-742

5. Forterre, P.: A hot topic: the origin of hyperthermophiles. Cell 85 (1996) 789-792

6. Philippe, H., Laurent, J.: How good are deep phylogenetic trees? Curr. Opin. Genet. Dev. 8 (1998) 616-623

7. Galtier, N., Tourasse, N., Gouy, M.: A nonhyperthermophilic common ancestor to extant life forms. Science 283 (1999) 220-221

8. Asai, T., Zaporojets, D., Squires, C., Squires, C.L.: An Escherichia coli strain with all chromosomal rRNA operons inactivated: complete exchange of rRNA genes between bacteria. Proc. Natl. Acad. Sci. USA 96 (1999) 1971-1976

9. Yap, W.H., Zhang, Z., Wang, Y.: Distinct types of rRNA operons exist in the genome of the actinomycete Thermomonospora chromogena and evidence for horizontal transfer of an entire rRNA operon. J. Bacteriol. 181 (1999) 5201-5209

10. Karlin, S., Campbell, A.M., Mrzek, J.: Comparative DNA analysis across diverse genomes. Annu. Rev. Genet. 32 (1998) 182-225

11. Doolittle, W.F.: Lateral genomics. Trends Cell Biol. 9 (1999) M5-8

12. Doolittle, W.F.: Phylogenetic classification and the universal tree. Science 284 (1999) 2124-2129

13. Bellgard, M.I., Itoh, T., Watanabe, H., Imanishi, T., Gojobori, T.: Dynamic evolution of genomes and the concept of genome space. Ann. NY Acad. Sci. 870 (1999) 293-300

14. Garcia-Vallv, S., Romeu, A., Palau, J.: Horizontal gene transfer in bacterial and archaeal complete genomes. Genome Res. 10 (2000) 1719-1725

15. Teichmann, S.A., Mitchison G.: Is there a phylogenetic signal in prokaryote proteins? J. Mol. Evol. 49 (1999) 98-107

16. Brown, J.R., Douady., C.J., Italia, M.J., Marshall, W.E., Stanhope, M.J.: Universal trees based on large combined protein sequence data sets. Nature Genet. 28 (2001) 281-285

17. Brochier, C., Bapteste, E., Moreira, D., Philippe, H.: Eubacterial phylogeny based on translational apparatus proteins. Trends Genet. 18 (2002) 1-5

18. Snel, B., Bork, P., Huynen, M.A.: Genome phylogeny based on gene content. Nature Genet. 21 (1999) 108-110

19. Tekaia, F., Lazcano, A., Dujon, B.: The genomic tree as revealed from whole proteome comparisons. Genome Res. 9 (1999) 550-557

20. Eisen, J.A.: Assessing evolutionary relationships among microbes from wholegenome analysis. Curr. Opin. Microbiol. 3 (2000) 475-480

21. Lin, J., Gerstein, M.: Whole-genome trees based on the occurrence of folds and orthologs: implications for comparing genomes on different levels. Genome Res. 10 (2000) 808-818

22. Baum, B.R.: Combining trees as a way of combining data sets for phylogenetic inference, and the desirability of combining gene trees. Taxon 41 (1992) 3-10

23. Ragan, M.A.: Phylogenetic inference based on matrix representation of trees. Mol. Phyl. Evol. 1 (1992) 53-58

24. Daubin, V., Gouy, M., Perrire, G.: A phylogenomic approach to bacterial phylogeny: evidence for a core of genes sharing common history. Genome Res. 12 (2002) 1080-1090

25. Perrière, G., Duret, L., Gouy, M.: HOBACGEN: database system for comparative genomics in bacteria. Genome Res. 10 (2000) 379-385 
26. Koski, L.B., Golding, G.B.: The closest BLAST hit is often not the nearest neighbor. J. Mol. Evol. 52 (2001) 540-542

27. Nelson, K.E., Clayton, R.A. Gill, S.R., Gwinn, M.L., Dodson, R.J., Haft, D.H., Hickey, E.K., Peterson, J.D., Nelson, W.C., Ketchum, K.A. et al.: Evidence of lateral gene transfer between Archaea and Bacteria from genome sequence of Thermotoga maritima. Nature 399 (1999) 323-329

28. Logsdon J.M., Faguy, D.M.: Thermotoga heats up lateral gene transfer. Curr. Biol. 9 (1999) R747-R751

29. Nesbo, C.L., L?Haridon, S. Stetter, K.O., Doolittle, W.F.: Phylogenetic analyses of two ?archaeal? genes in Thermotoga maritima reveal multiple transfers between archaea and bacteria. Mol. Biol. Evol. 18 (2001) 362-375

30. Thompson, J.D., Higgins, D.G., Gibson, T.J.: CLUSTAL W: improving the sensitivity of progressive multiple sequence alignment through sequence weighting, position specific gap penalties and weight matrix choice. Nucleic Acids Res. 22 (1994) 4673-4680

31. Castresana, J.: Selection of conserved blocks from multiple alignments for their use in phylogenetic analysis. Mol. Biol. Evol. 17 (2000) 540-552

32. Guindon, S., Gascuel, O.: A simple, fast, and accurate algorithm to estimate large phylogenies by maximum likelihood. Syst. Biol. 52 (2003) 696-704

33. Gascuel, O.: BIONJ: an improved version of the NJ algorithm based on a simple model of sequence data. Mol. Biol. Evol. 14 (1997) 685-695

34. Jones, D.T., Taylor, W.R., Thornton, J.M.: The rapid generation of mutation data matrices from protein sequences. Comput. Applic. Biosci. 8 (1992) 275-282

35. Felsenstein, J.: PHYLIP ? Phylogeny inference package (Version 3.2). Cladistics 5 (1989) 164-166

36. Strimmer, K., von Haeseler, A.: Quartet puzzling: a quartet maximum likelihood method for reconstructing tree topologies. Mol. Biol. Evol. 13 (1996) 964-969

37. Liu, F.G., Miyamoto, M.M., Freire, N.P., Ong, P.Q., Tennant, M.R., Young, T.S., Gugel, K.F.: Molecular and morphological supertrees for eutherian (placental) mammals. Science 291 (2001) 1786-1789

38. Swofford, D. L.: PAUP*. Phylogenetic Analysis Using Parsimony (*and Other Methods). Version 4. Sinauer Associates, Sunderland, Massachusetts (2003)

39. Gupta, R.S.: The phylogeny of proteobacteria: relationships to other eubacterial phyla and eukaryotes. FEMS Microbiol. Rev. 24 (2000) 367-402

40. Kreil, D.P., Ouzounis, C.A.: Identification of thermophilic species by the amino acid compositions deduced from their genomes. Nucleic Acids Res. 29 (2001) 16081615 